See discussions, stats, and author profiles for this publication at: https://www.researchgate.net/publication/285202536

Gellan gum microspheres crosslinked with trivalent ion: Effect of polymer and crosslinker concentrations on drug release and mucoadhesive properties

Article in Drug Development and Industrial Pharmacy · November 2015

DOI: 10.3109/03639045.2015.1125915

CITATIONS

8

3 authors:

Fernanda Isadora Boni

São Paulo State University

8 PUBLICATIONS 35 CITATIONS

SEE PROFILE

Beatriz Stringhetti Ferreira Cury

28 PUBLICATIONS 375 CITATIONS

SEE PROFILE

Some of the authors of this publication are also working on these related projects:

Evaluation of peptides release using a natural rubber latex biomembrane as a carrier View project

pH RESPONSIVE HYDROGELS AS POTENTIAL DRUG DELIVERY SISTEM FOR BEVACIZUMAB ADMINISTRATION View project 


\title{
Gellan gum microspheres crosslinked with trivalent ion: effect of polymer and crosslinker concentrations on drug release and mucoadhesive properties
}

\author{
Fernanda Isadora Boni, Fabíola Garavello Prezotti \& Beatriz Stringhetti \\ Ferreira Cury
}

To cite this article: Fernanda Isadora Boni, Fabíola Garavello Prezotti \& Beatriz Stringhetti Ferreira Cury (2016) Gellan gum microspheres crosslinked with trivalent ion: effect of polymer and crosslinker concentrations on drug release and mucoadhesive properties, Drug Development and Industrial Pharmacy, 42:8, 1283-1290, DOI: 10.3109/03639045.2015.1125915

To link to this article: http://dx.doi.org/10.3109/03639045.2015.1125915

Accepted author version posted online: 29

Nov 2015.

Published online: 29 Jan 2016.

Submit your article to this journal $\asymp$

凹ll Article views: 56

View related articles $\sqsubset$

View Crossmark data $₫$ 


\title{
Gellan gum microspheres crosslinked with trivalent ion: effect of polymer and crosslinker concentrations on drug release and mucoadhesive properties
}

\author{
Fernanda Isadora Boni, Fabíola Garavello Prezotti and Beatriz Stringhetti Ferreira Cury
}

Graduate Program in Pharmaceutical Sciences, Department of Drugs and Pharmaceuticals, School of Pharmaceutical Sciences, São Paulo State University - UNESP, Araraquara, SP, Brazil

\begin{abstract}
Gellan gum microspheres were obtained by ionotropic gelation technique, using the trivalent ion $\mathrm{Al}^{3+}$. The percentage of entrapment efficiency ranged from 48.76 to $87.52 \%$ and $2^{2}$ randomized full factorial design demonstrated that both the increase of polymer concentration and the decrease of crosslinker concentration presented a positive effect in the amount of encapsulated drug. Microspheres size and circularity ranged from 700.17 to $938.32 \mu \mathrm{m}$ and from 0.641 to $0.796 \mu \mathrm{m}$, respectively. The increase of polymer concentration (1-2\%) and crosslinker concentration (3-5\%) led to the enlargement of particle size and circularity. However, the association of increased crosslinker concentration and reduced polymer content made the particles more irregular. In vitro and ex vivo tests evidenced the high mucoadhesiveness of microspheres. The high liquid uptake ability of the microspheres was demonstrated and the $\mathrm{pH}$ variation did not affect this parameter. Drug release was $\mathrm{pH}$ dependent, with low release rates in acid $\mathrm{pH}(42.40 \%$ and $44.93 \%)$ and a burst effect in phosphate buffer $\mathrm{pH}$ (7.4). The Weibull model had the best correlation with the drug release data, demonstrating that the release process was driven by a complex mechanism involving the erosion and swelling of the matrix or by non-Fickian diffusion.
\end{abstract}

\section{ARTICLE HISTORY}

Received 10 August 2015 Revised 23 October 2015 Accepted 22 November 2015 Published online 29 January 2016

\section{KEYWORDS}

Colonic drug delivery; dissolution test; ionotropic gelation; mucoadhesion; multiparticulate system

\section{Introduction}

Oral dosage forms exploit the absorptive capacity of the gastrointestinal tract (GIT) and their wide use in the therapeutics is due to patient convenience, dose flexibility, safety, and low cost compared to other routes of administration ${ }^{1,2}$. After the administration of a conventional dosage form, the drug is released relatively quickly and several side effects and/or adverse reactions may appear ${ }^{3,4}$.

The targeting of drugs to a specific organ or tissue is a great challenge in research and development of new oral controlled drug delivery systems. To achieve the site of action or absorption, the drug has to transpose many biological barriers such as others organs, rough environments, tissues, or intracellular compartments, where it may be degraded or even affect sites that are not involved in the pathological process ${ }^{5}$.

Among the several organs along the GIT, the colon arouses particular interest for the treatment of local pathologies, such as inflammatory diseases and colonic cancer, as well as systemic pathologies, since it presents an extended transit time, reduced proteolytic activity and $\mathrm{pH}$ near to neutrality ${ }^{6}$. By these characteristics, the colon becomes a promising site for drug release, mainly those with permeability and/or stability issues in upper portions of the $\mathrm{GIT}^{7,8}$.

A wide variety of synthetic, semi-synthetic, or natural polymers find application in the development of controlled drug release systems ${ }^{9-11}$. The increasing interest in the development of drug carrier systems based on natural hydrophilic polymers is justified because they are low cost, stable, biocompatible, nontoxic, have good gelling properties, and present a variety of structures able to be chemically and/or physically modified, conferring several properties that can be modulated according to specific needs ${ }^{12-14}$.

Microencapsulation technology allows reaching different goals such as protect the drug from hostile conditions and incompatibilities, mask unpleasant taste and control the drug release rates in order to maximize therapeutic effects and minimize systemic side effects $^{15,16}$. Microcapsules and microspheres can be obtained using many simple and low-cost materials and techniques, and their size ranges from 1 to $1000 \mu \mathrm{m}^{17,18}$.

In fact, these multiparticulate systems have several advantages over single-unit dosage forms, such as gastric emptying time more predictable and less dependent of the nutritional state; more uniform distribution of the drug on the GIT surface with reduced risk of local irritation ${ }^{19,20}$. For obtaining microspheres, various methods are proposed, like emulsification-solvent removal, coacervation, ionotropic gelation, polymerization, spray drying, and supercritical fluid atomization. To select the most appropriate method, it must be considered the type of the drug that will be encapsulated, the release mechanism, and the desired application ${ }^{18,21}$.

In recent years, the development of hydrogel microspheres from polysaccharides by ionotropic gelation has been focus of important researches, because changes in polymer concentration and crosslinking degree can lead to the production of systems with different drug release profiles for specific goals ${ }^{22,23}$.

Gellan gum (GG) is a hydrophilic and anionic exopolysaccharide obtained aerobically from the bacteria Sphingomonas elodea and composed by repeated units of glucose, glucuronic acid, and rhamnose in 2:1:1 molecular ratio, and two acetyl substituents,

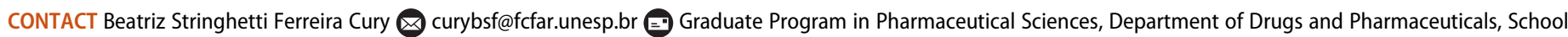
of Pharmaceutical Sciences, São Paulo State University - UNESP, Rodovia Araraquara-Jaú, Km 1, Araraquara, SP 14801-902, Brazil 
acetate, and glycerate, linked on glucose residue adjacent of the glucuronic acid ${ }^{24,25}$.

GG is widely used as an additive in food industry; at low concentrations, it forms gels and it is biocompatible and biodegradable. In aqueous solution, the gelation of GG is accompanied by the chains conformational transition from random coils to doublehelical conformation and then the double-helical arrangement form ordered junction zones, resulting in a three-dimensional network $^{11,26}$. In the presence of cations, the ionotropic gelation occurs, in which polymer negative groups interact with a divalent or multivalent counter ion, building a stronger and dense hydrogel network ${ }^{27,28}$.

These characteristics make GG an interesting material for pharmaceutical applications ${ }^{22,29}$. Several drug delivery systems based on GG, such as in situ momethasone furoate nasal gel ${ }^{30}$, in situ gelling terbinafine hydrochloride ophtalmic nanoemulsion ${ }^{31}$, and GG tablets with metronidazole ${ }^{32}$ were developed. The development of hydrogel microspheres based on GG crosslinked with mono and divalent cations, mainly $\mathrm{Ca}^{2+}$, has been exploited to obtain different drug delivery systems ${ }^{22,24}$.

Trivalent cation $\left(\mathrm{Al}^{3+}\right)$ have been evaluated for microspheres production because the crosslinking with trivalent ions can be advantageous by enabling a faster crosslinking reaction due to their extra positive charge, when compared, for example, with calcium ions. Each molecule of aluminum is able to conjugate with a higher number of sites of the polysaccharides. Therefore, the use of a low concentration of crosslinking solution may allow the faster formation of a more rigid hydrogel, reducing the reaction time and consequently reducing the risk of drug solubilization or degradation in the crosslinking solution, providing a more efficient drug encapsulation and drug release control ${ }^{33-35}$.

Maiti and coworkers reported the preparation of GG microspheres crosslinked with aluminum ions and glutaraldehyde for prolonged release of glipizide, studying the influence of the dual crosslinking (ionic and covalent) in microspheres characteristics and drug release profiles ${ }^{23}$. Prezotti and coworkers (2014) developed microspheres of GG and pectin blends, crosslinked with $\mathrm{Al}^{3+}$, demonstrating the effective reduction of release rates of ketoprofen (KP) in simulated gastric media ${ }^{36}$

Traditional methods of performing experiments involve material and time spending, especially when new formulations are developed. The factorial design technique is an efficient method to indicate significant effects of variables and their interactions. The use of this technique helps optimizing the samples preparation and analysis of the process, minimizing the amount of time and materials spent during the development ${ }^{37,38}$.

In this paper, GG microspheres containing KP as model drug were prepared by ionotropic gelation using aluminum chloride as crosslinker. The physicochemical characterization included analyses of size, shape, surface morphology and internal structure, swelling ability, and entrapment efficiency (EE\%). The influence of the variables polymer and crosslinker concentrations on particle size, shape, and $\mathrm{EE} \%$ was evaluated by a $2^{2}$ randomized full factorial design. The mucoadhesive ability of microspheres was assessed through in vitro and ex vivo analysis, and the drug release profile was evaluated in media that simulate the $\mathrm{pH}$ variation of the GIT.

\section{Materials and methods}

\section{Materials}

Low acyl GG (200-300 KDa) was kindly provide by CP Kelco (Kelcogel $^{\circledR}$ CG-LA); KP (batch \# 09072223) was obtained from Zhejiang Jiuhzou Pharmaceutical Co. (Taizhou, China); aluminum
Table 1. Composition of the beads formulations and codes for the $2^{2}$ randomized factorial design employed.

\begin{tabular}{lccc} 
Sample & $\begin{array}{c}\text { GG concentration } \\
(\%)\left(\mathrm{X}_{1}\right)\end{array}$ & $\begin{array}{c}\mathrm{Al}^{3+} \\
\text { concentration } \\
(\%)\left(\mathrm{X}_{2}\right)\end{array}$ & $\begin{array}{c}\text { Drug } \\
\text { concentration } \\
(\%)\end{array}$ \\
\hline G1Al3 & $1.0(-1)$ & $3.0(-1)$ & 1.0 \\
G1Al5 & $1.0(-1)$ & $5.0(+1)$ & 1.0 \\
G2Al3 & $2.0(+1)$ & $3.0(-1)$ & 1.0 \\
G2Al5 & $2.0(-1)$ & $5.0(+1)$ & 1.0 \\
G2Al3A-C & 2.0 & 3.0 & 0.0 \\
G2Al5A-C & 2.0 & 5.0 & 0.0 \\
\hline
\end{tabular}

chloride from Vetec; Mucin type II and Total Protein Kit Micro Lowry, Peterson's Modification were purchased from Sigma Aldrich ${ }^{\circledR}$ (St. Louis, MO). All other materials used were of analytical grade and obtained from commercial suppliers.

\section{Factorial design}

A $2^{2}$ randomized full factorial design was used in this study to evaluate the effects of the variables GG concentration (X1) and crosslinker concentration (X2) on microspheres size, shape and EE\%. The independent variables levels and respective values of the experimental design are given in Table 1.

\section{Preparation of microspheres}

Microspheres were prepared by ionotropic gelation method. Aqueous dispersions of GG $(1.0 \%$ or $2.0 \% \mathrm{w} / \mathrm{v})$ at $\mathrm{pH} 4.0$ were prepared under magnetic stirring at $60^{\circ} \mathrm{C}$. KP $(1 \% \mathrm{w} / \mathrm{v})$ was added under constant stirring, until complete homogenization. Microspheres without drug were prepared as control.

Polymer dispersions were dripped into the cooled $\left(4^{\circ} \mathrm{C}\right)$ crosslinking solution containing aluminum chloride $3 \%$ or $5 \%$, using syringe and flat-tipped needles (23G), under constant magnetic stirring. The microspheres were kept under constant stirring for $20 \mathrm{~min}$ to complete the crosslinking reaction. The microspheres were separated by filtration, washed with distilled water, and dried at room temperature until constant weight.

Samples were labeled according to the GG concentration ( $\mathrm{G} 1=1 \%$ and $\mathrm{G} 2=2 \%$ ) and crosslinking solution concentration $(\mathrm{Al} 3=3 \%$ and $\mathrm{Al} 5=5 \%$ of $\mathrm{AlCl} 3)$. Control microspheres without drug were labeled without suffix $C$ (Table 1).

\section{Evaluation and characterization of microspheres}

\section{Particles size and shape analysis}

Microspheres were analyzed on a stereoscope (Leica MZ APO ${ }^{\circledR}$ ), and images were captured using Motic Images Advance 2.0 program. Microspheres size and shape were measured using an image analysis program (Motic Advance Images 3.2). The average diameter and circularity of 100 microspheres of each formulation were calculated. To evaluate samples polydispersity, the Span index was determined based on size distribution data, following Equation (1):

$$
\text { Span }=(\text { D90 }- \text { D10 }) / \text { D50 }
$$

where D90, D10, and D50 are the diameters $(\mu \mathrm{m})$ determined for 90 th, 10th, and 50th percentile, respectively.

\section{Scanning electron microscopy}

The microspheres surface and internal structure of microspheres were analyzed by field emission gun scanning electron microscopy 
(FEG-SEM; JEOL JSM-7500F, Japan). To evaluate the internal structure, microspheres were frozen with liquid nitrogen and after fractured. Samples were attached to the sample holder with a double-side adhesive tape, and photomicrographs at different magnifications were taken.

\section{Entrapment efficiency}

To determine the EE\%, a known mass of dried microspheres was allowed to swell in $0.1 \mathrm{ml}$ of phosphate buffer $(\mathrm{pH}$ 7.4) during $5 \mathrm{~min}$ and then crushed inside the centrifuge tube. The microspheres were added by absolute ethanol $(10 \mathrm{ml})$ to dissolve the drug and kept under stirring for $1.5 \mathrm{~h}$, at room temperature. Tubes were well sealed to avoid solvent evaporation. After, samples were centrifuged at $2000 \mathrm{rpm}(5 \mathrm{~min})$ to precipitate polymeric debris, and the amount of drug in the supernatant was quantified on a UV-Vis spectrophotometer (Hewlett Packard-Kayak XA), at $254 \mathrm{~nm}$. Tests were performed in triplicate, and the EE\% was calculated according Equation (2):

$$
\% \mathrm{EE}=(\mathrm{AA} / \mathrm{TA}) * 100
$$

where TA was the total amount of drug added, and AA was the quantified amount of drug.

\section{Liquid uptake study}

Swelling study was performed on an Enslin device with different media to simulate the variation throughout the GIT: simulated gastric fluid $(0.1 \mathrm{~N} \mathrm{HCl}, \mathrm{pH} 2.0)$, simulated intestinal fluid $(\mathrm{pH} 7.4)$, and simulated colonic fluid ( $\mathrm{pH} 6.0$ ), all of them without enzymes. The volume of media uptake by the sample was measured at predetermined time intervals $(1,2,5,10,15,30,60$, and 90 min). The tests were performed in triplicate and results were expressed as percentage of swelling (\%S), according to Equation (3):

$$
\% S=V / m \times 100
$$

where $m$ is the initial mass of microspheres $(\mathrm{g}) ; V=$ volume $(\mathrm{mL})$ of media absorbed; $\% S=$ percentage of swelling (\%).

\section{In vitro analysis of the mucoadhesive properties}

The mucoadhesiveness was analyzed by studying the adsorption of mucin on the microspheres ${ }^{3}$. Mucin solutions were prepared at different concentrations $(50,100,150$, and $200 \mu \mathrm{g} / \mathrm{ml})$, and $20 \mathrm{mg}$ of microspheres were dispersed in these solutions for $1 \mathrm{~h}$ at $37^{\circ} \mathrm{C}$. Then, the dispersions were centrifuged at $3000 \mathrm{rpm}(2 \mathrm{~min})$, and the supernatant was used to quantify the free mucin content by colorimetric method, using a Lowry protein assay modified by Peterson 39,40 . For the determination of free mucin in the supernatant, an UV-Vis spectrophotometer at $749 \mathrm{~nm}$ and reagents from Total Protein Kit, Micro Lowry, Peterson's Modification (Sigma-Aldrich ${ }^{\circledR}$, St. Louis, MO) were used $^{36}$. For the mucoadhesive property analysis, samples containing high amount of GG (2\%) were selected, since they presented higher circularity and $\mathrm{EE} \%$.

\section{Ex vivo mucoadhesion evaluation}

The ex vivo mucoadhesion test was performed as described by Prezotti et al. using porcine intestinal tissue. The method was based on the procedure purposed by Rao and Bur ${ }^{41}$. Briefly, pieces of fresh tissue $(4 \mathrm{~cm} \times 4 \mathrm{~cm})$ were opened longitudinally and washed using saline solution (0.9\%) and attached to the inclined plastic support $\left(30^{\circ} \mathrm{C}\right)$ of the device. The microspheres $(n=30)$ were placed on the tissue surface, allowing a 20-min contact time with the mucous layer. After this, phosphate buffer pH 6.0 was used to rinse the tissue for $5 \mathrm{~min}$ at a rate of $30 \mathrm{ml} / \mathrm{min}$. The percentage of mucoadhesion was calculated from the difference between the number of microspheres attached to the biological surface at the beginning and at the end of the test.

\section{Dissolution test and analysis of drug release mechanism}

This analysis was performed on a Hanson Dissolution Test Station SR8-Plus (Chastworth, CA) equipped with USP apparatus 1 (basket) at $50 \mathrm{rpm}$. The experiment was conducted using media with different $\mathrm{pH}$ values at $37{ }^{\circ} \mathrm{C}$ : simulated gastric media $(900 \mathrm{~mL}$ of $0.1 \mathrm{~N} \mathrm{HCl} \mathrm{pH} 1.2$ with sodium lauryl sulfate $0.75 \%$ ) during $120 \mathrm{~min}$ and enteric media $(900 \mathrm{~mL}$ of phosphate buffer $\mathrm{pH}$ 7.4). At predetermined time intervals, aliquots of $3 \mathrm{ml}$ were withdrawn and immediately replaced with fresh dissolution media. The amount of drug released was quantified using an UV-Vis spectrophotometer at $258 \mathrm{~nm}$ and $260 \mathrm{~nm}$, for gastric and enteric $\mathrm{pH}$, respectively. The experiment was conducted with raw KP and samples G2Al3 and G2Al5, once they presented the highest values of EE\%. The tests were performed in triplicate, with a mass of microspheres containing $100 \mathrm{mg}$ of KP.

Drug release data were fitted with different mathematical models (Korsmeyer-Peppas, Higuchi, First-order, Hixson-Crowell and BakerLonsdale) to determine the mechanism of drug release.

\section{Statistical analysis}

One-way analysis of variance (ANOVA) followed by Tukey's test was used to evaluate significant differences with a significance level of $5 \%$.

\section{Results}

Evaluation and characterization of microspheres

\section{Particles size and shape analysis}

The diameter of the microspheres ranged from 700.17 to $938.32 \mu \mathrm{m}$ (Table 2) with sample G1Al3 presenting the smallest mean diameter $(700.17 \mu \mathrm{m})(p<0.05)$. GG microspheres presented circularity values between 0.641 and 0.796 (Table 2). Samples showed a monodispersed and unimodal size distribution, with Span index between 0.08 and 0.18 (Table 2).

\section{Scanning electron microscopy}

The microspheres SEM photomicrographs showed very irregular surfaces and KP crystals in the internal structure (Figure 1).

\section{Entrapment efficiency}

The microspheres EE\% ranged from 48.76 to $87.52 \%$ (Table 2).

\section{Liquid uptake study}

The results demonstrated the high liquid uptake ability of the samples, with values ranging from $216.2 \%$ to $303.3 \%$ in media with $\mathrm{pH} 1.2$; from $248.3 \%$ to $255.2 \%$ in media with $\mathrm{pH} 6.0$, and from $195.5 \%$ to $245.8 \%$ in media with $\mathrm{pH} 7.4$ (Table 2 ). 
Table 2. Values of circularity, size $(\mu \mathrm{m})$, span index, EE\%, and swelling (\%S) of GG beads obtained through ionotropic gelation and Weibull coefficients from drug release studies (mean \pm SD).

\begin{tabular}{|c|c|c|c|c|c|c|c|c|c|c|c|}
\hline \multirow[b]{3}{*}{ Sample } & \multirow[b]{3}{*}{ Circularity } & \multirow[b]{3}{*}{ Diameter $(\mu \mathrm{m})$} & \multirow[b]{3}{*}{ Span } & \multirow[b]{3}{*}{$\% \mathrm{EE}$} & \multirow{2}{*}{\multicolumn{3}{|c|}{$\% \mathrm{~S}$}} & \multicolumn{4}{|c|}{ Weibull coefficients } \\
\hline & & & & & & & & \multicolumn{2}{|c|}{ pH 1.2} & \multicolumn{2}{|c|}{$\mathrm{pH} 7.4$} \\
\hline & & & & & $\mathrm{pH} 1.2$ & $\mathrm{pH} 6.0$ & pH 7.4 & $r^{2}$ & $b$ & $r^{2}$ & $b$ \\
\hline G1Al3 & $0.739 \pm 0.059$ & $700.17 \pm 94,86$ & 0.13 & $68.79 \pm 3.36$ & $222.03 \pm 23.14$ & $249.58 \pm 16.95$ & $195.49 \pm 15.37$ & - & - & - & - \\
\hline G1Al5 & $0.641 \pm 0.119$ & $812.46 \pm 103.59$ & 0.18 & $48.76 \pm 2.56$ & $216.19 \pm 36.01$ & $248.32 \pm 9.63$ & $242.93 \pm 5.82$ & - & - & - & - \\
\hline G2Al3 & $0.679 \pm 0.079$ & $866.52 \pm 55.67$ & 0.08 & $87.52 \pm 8.31$ & $303.03 \pm 44.37$ & $255.22 \pm 15.30$ & $245.85 \pm 5.47$ & 0.9898 & 1.22 & 0.9948 & 0.8819 \\
\hline G2Al5 & $0.796 \pm 0.034$ & $938.32 \pm 43.79$ & 0.14 & $82.34 \pm 2.90$ & $261.31 \pm 56.85$ & $253.86 \pm 8.29$ & $204.18 \pm 21.99$ & 0.9546 & 1.943 & 0.9967 & 2.220 \\
\hline
\end{tabular}
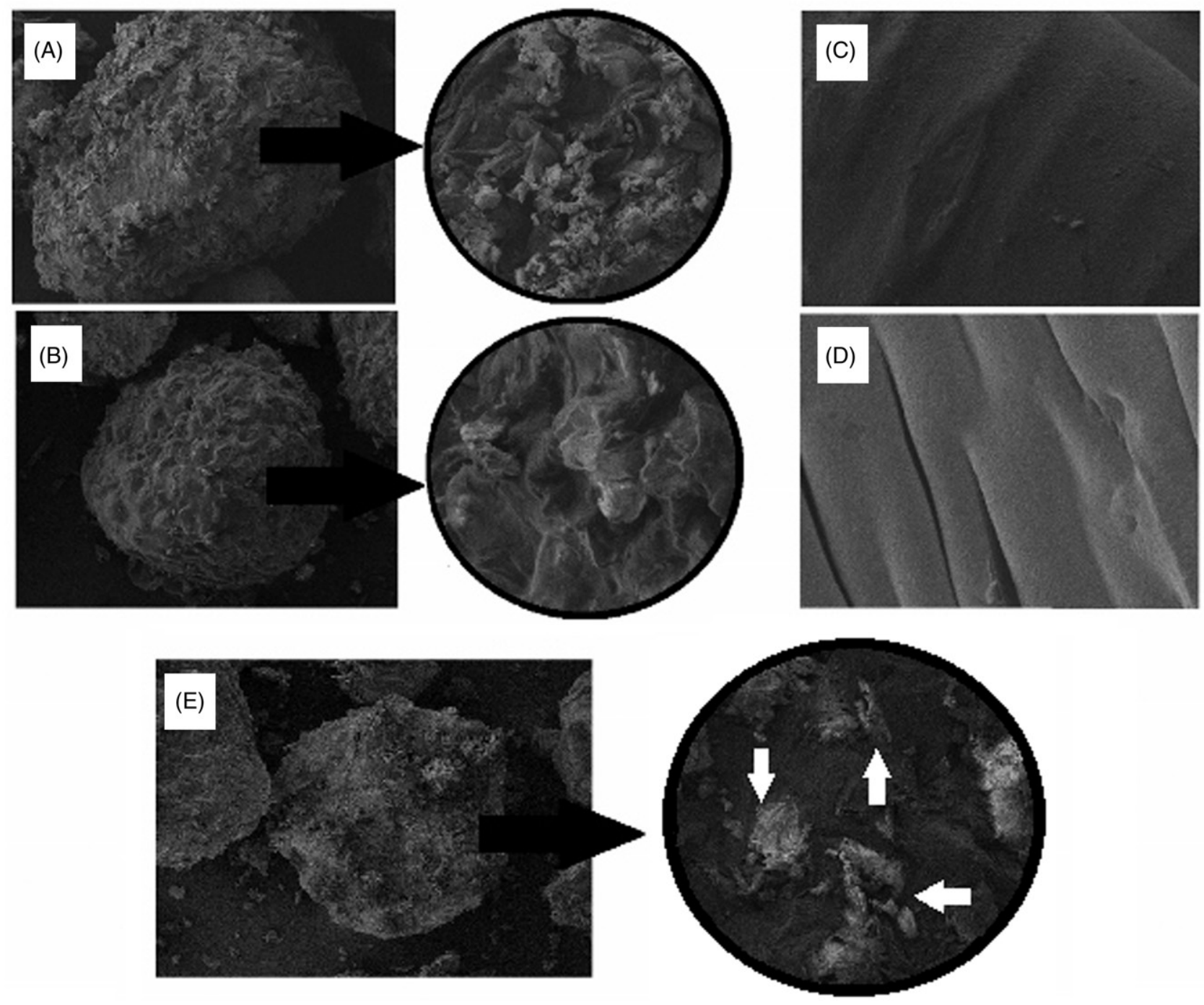

Figure 1. Photomicrographs of a) G2Al3; (b) G2Al5 evidencing the irregular surface ( $100 \times$ and $1000 \times$ ); (c) G2Al3-C; (d) G2Al5-C evidencing the smooth surface (1000 $\times$ ); (e) G1Al3 evidencing the internal structure $(50 \times$ and $1000 \times)$.

\section{In vitro analysis of the mucoadhesive properties}

At the in vitro evaluation, the increase in mucin concentration increased the amount of adsorbed mucin (Figure 2). For G2Al3, the mucin adsorbed ranged from 408.97 to $1778.84 \mu \mathrm{g}$, and for G2Al5 from 340.45 to $1687.67 \mu \mathrm{g}$ (Figure 2).

\section{Ex vivo mucoadhesion evaluation}

The microspheres presented $100 \%$ of mucoadhesive capacity at the end of the ex vivo test.

\section{Dissolution test and analysis of drug release mechanism}

The dissolution rates of free KP and from the microspheres are presented in Figure 3.

After 120 min in acid $\mathrm{pH}$, about $72 \%$ of free KP was dissolved, and the dissolution was completed in pH 7.4 after 180 min (Figure 3). In the first 120 min in acid $\mathrm{pH}, \mathrm{G} 2 \mathrm{Al} 3$ and G2Al5 released $42.40 \%$ and $44.93 \%$ of the drug, respectively. The dissolution rate in acid media was lower than the free KP (about 1.64 times), demonstrating the ability of the system in controlling the drug release rates. In media with $\mathrm{pH} 7.4$, a burst effect was observed, so that $87.23 \%$ and $90.75 \%$ 


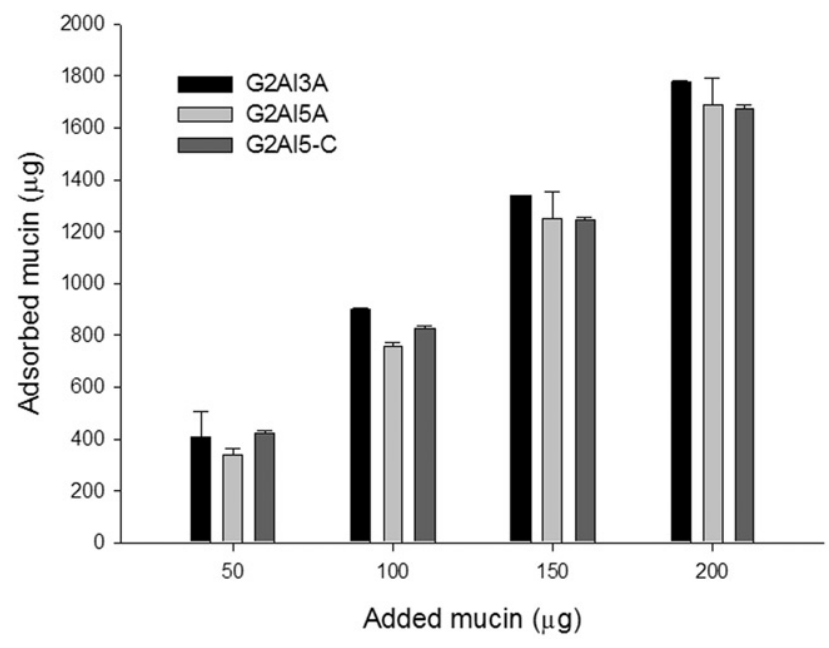

Figure 2. Relation between the amount of mucin added and the mucin adsorbed on different $G G$ beads.

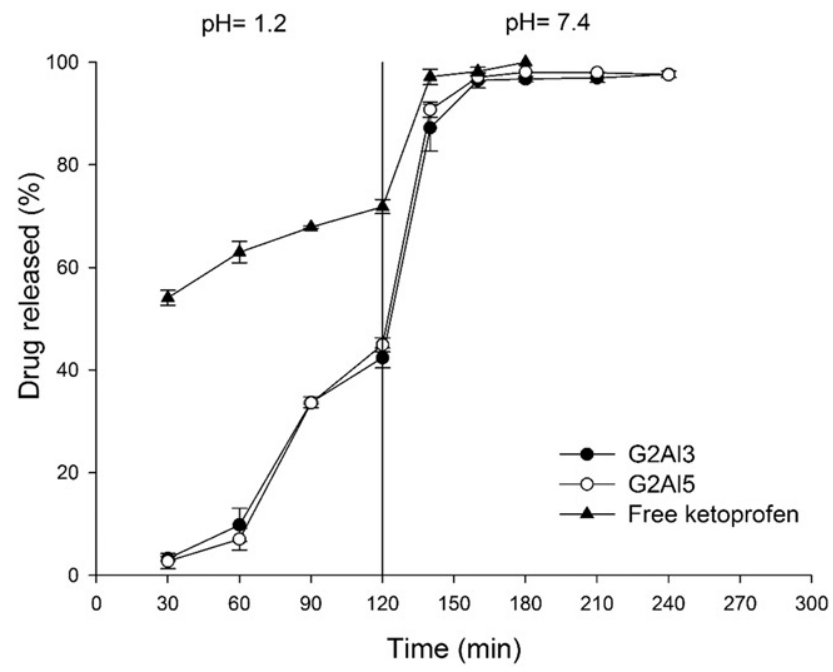

Figure 3. Drug release profile from GG beads.

of the drug was released in $140 \mathrm{~min}$, and $100 \%$ of the drug was released after 240 min for G2Al3 and G2Al5 samples.

The release profiles were analyzed using mathematical models in order to describe the mechanism of drug release from the microspheres. According to the correlation coefficient $\left(r^{2}\right)$, the model that better describes the release is the Weibull model (Table 1).

\section{Discussion}

\section{Preparation of microspheres}

GG microspheres were prepared by ionotropic gelation in which negatively charged GG interacted with aluminum ions. It was observed that concentrations of GG below 1\% did not allow the formation of spherical particles. The low viscosity of the polymer dispersion and the superficial tension could not counteract the impact and drag forces that droplets suffer when enter in contact with the crosslinking solution, so the droplets did not penetrate correctly on the solution and nonspherical particles were formed ${ }^{42,43}$. When the polymer concentration was increased $(2 \%)$, the viscosity of the dispersion was improved, so that the droplets were more resistant against the drag forces and spherical microspheres were built.

\section{Characterization of microspheres}

Hydrogel droplets are formed during the dripping using a syringe and a flat-tipped needle, and these droplets should be as regular as possible and overcome the impact and drag forces with the surface of the crosslinking solution in order to maintain the spherical shape after the crosslinking reaction. The higher viscosity of the dispersions with higher polymer concentration allowed a greater resistance against deformation during the dripping process, and when the number of $\mathrm{Al}^{3+}$ ions was increased in the gelling bath, a faster crosslinking occurred, originating a more rigid polymer network that kept the spherical shape ${ }^{42-44}$.

From the factorial design, it can be concluded that both polymer and crosslinker concentration had significant effects on particles size $(p<0.05)$. The increase in polymer concentration from 1 to $2 \%$ led to an increase in particle size. The high polymer concentration forms a more viscous dispersion and thus bigger droplets during the dripping are formed, originating microspheres of increased diameter $^{36,45}$. The increase of crosslinker concentration from 3 to $5 \%$ also promoted an increase in particle size $(p<0.05)$. In the presence of a large number of $\mathrm{Al}^{3+}$ ions, a more branched network can be built, enlarging the particle size $\mathrm{s}^{36}$.

Factorial design results also showed that polymer concentration had a significant effect on particles circularity as well as a significant interaction between polymer and crosslinker concentration $(p<0.05)$. The low polymer content $(1 \%)$ associated with the high crosslinker concentration (5\%) decreased the particles circularity and the sample G1Al5 presented the lowest circular degree (0.641). On the other hand, the high polymer content (2\%) associated with the high crosslinker concentration (5\%) improved the microspheres circularity, and the sample G2Al5 showed the highest circularity (0.796; $p<0.05)$.

From SEM photomicrographs, it was possible to observe that the increase of GG and crosslinking concentrations, as well as the presence of drug, increased the surface irregularity. Control samples without drug presented a smooth and homogeneous surface, and the increase of crosslinker concentration caused more indentation. The photomicrographs from the internal structure showed that KP crystals distributed inside the polymer matrix.

Microspheres presented high percentages of drug encapsulation. From the factorial design, it can be concluded that both polymer and crosslinker concentration presented significant effects on EE\% $(p<0.05)$. Polymer concentration presented a positive effect in the amount of encapsulated drug, so that at high GG content (2\%), the $\mathrm{EE} \%$ was increased. In the presence of higher amount of polymer chains, the network produced by the crosslinking with $\mathrm{Al}^{3+}$ ions is more able to entrap the drug inside the matrix ${ }^{46}$. However, crosslinker concentration showed a negative effect on $\mathrm{EE} \%$ that decreased when aluminum chloride concentration was higher (5\%). There was a significant interaction effect between the two selected variables, and the condition that allowed the best $\mathrm{EE} \%$ (87.52\%) was the presence of high polymer and low crosslinker concentrations.

\section{Liquid uptake study}

The swelling process of a polymeric material occurs at molecular level and involves the diffusion of molecules from a liquid into the polymer network. This diffusion is possible due to the mobility of local segments of the polymer chains, which accommodate the liquid molecules. However, it is known that the ionic crosslinking 
process immobilizes the polymer chains, and consequently the swelling ability of the polymeric system can be reduced ${ }^{47,48}$.

The liquid uptake ability is an important feature of drug delivery systems since the water absorption is the first step for the drug release process, for further matrix swelling and/or diffusion of the drug. However, the drug dissolution and the matrix erosion can also impel the drug release process ${ }^{49,50}$. This ability is also important because it can ensure the access of bacterial enzymes that act in polymer matrix degradation and the liquid uptake can assist in the mucoadhesion process, as the absorption of liquid provides polymer chains relaxation and makes them available to interact with mucus layer $^{51-53}$.

All microspheres presented a non $\mathrm{pH}$ sensitive liquid uptake behavior, since there were no significant differences in the percentage of swelling $(\% \mathrm{~S})$ between the different media for all samples $(p>0.05)$.

\section{Mucoadhesion tests}

Mucoadhesion is an important property for multiparticulate systems because it may prolong the residence time in the absorption site and promote a closer contact with the epithelial barrier, increasing drug permeability and absorption ${ }^{54-56}$.

Colon has a low mucus turnover rate compared to the stomach and the small intestine and a slow motility; these features facilitate the contact of the polymeric system with the glycoproteins which are the structural components of mucus and primarily responsible for the adhesive interaction and enable the consolidation phase of the mucoadhesion mechanism ${ }^{57,58}$.

The mucin adsorption values were about four times higher than those observed by Dhawan and coauthors in a study with chitosan microspheres and very similar to the results obtained by Prezotti et al. with microspheres of GG/pectin blends. The adsorption ability was not influenced by crosslinker concentration and drug presence $(p>0.05)$. Considering that the mucin solutions had $\mathrm{pH} 4.79$, higher than the pKa of the GG (pKa 3.5) and mucin (pKa 2.6), both should be negatively charged due to ionization of the carboxyl groups. Thus, the electron theory does not explain the mucoadhesion process and the adsorption of mucin to the microspheres. Possibly, the electrostatic repulsion due to negative charges of the polymers made the chains farther from each other, facilitating the interaction and the bond formation with mucin glycoproteins chains

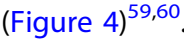

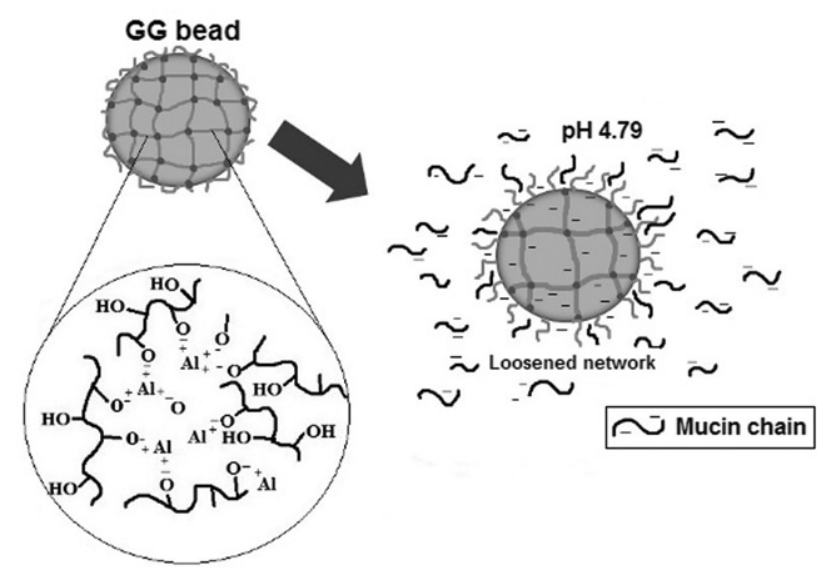

Figure 4. Schematic illustration of the mucoadhesion process by electrostatic repulsion of the polymers chains and interaction with mucin.
In the ex vivo mucoadhesion test, all microspheres from all samples were strongly attached to the porcine mucosa. This result corroborates with the in vitro mucoadhesion results, evidencing the high mucoadhesive ability of the microspheres developed in this study.

\section{Dissolution test and analysis of drug release mechanism}

The dissolution results demonstrated that the crosslinking reaction allowed the effective control of drug release in acid media but the crosslinking degree did not affect significantly the drug release rates $(p>0.05)$. The increase in the $\mathrm{pH}$ should increase the dissociation of polymer carboxyl groups, resulting in a more dilated and hydrophilic polymer network, favoring the entry of liquid into the matrix and the further dissolution and release of the drug. The set of results evidenced the $\mathrm{pH}$-dependent drug release behavior with reduced release rates in acid media $^{22,35}$

The Weibull model that better described the release mechanism expresses the cumulative amount of drug at the dissolution medium at a certain time and can be adjusted to different dissolution profiles ${ }^{61}$. In this model, the $b$ exponent value indicates the drug release mechanism through the polymer matrix. Values of $b<0.75$ indicate Fickian diffusion, values of $0.75<b<1.0$ indicate a non-Fickian diffusion, and values of $b>1.0$ indicate a complex drug release mechanism that involves diffusion, swelling, and/or erosion of the matrix ${ }^{14,62}$. As seen in Table 1 , the $b$ coefficient values were higher than 1 in both medium, except for the G2AI3, indicating that the drug release is driven by a complex mechanism involving erosion and swelling of the matrix. The G2Al3 sample showed a value of $b<1$ at $\mathrm{pH} 7.4$, indicating that the release mechanism of the drug from the matrix followed a non-Fickian diffusion, in which the drug diffusion is governed by the matrix swelling and the polymer chains relaxation. Therefore, the change in the media $\mathrm{pH}$ value altered the drug dissolution profile but did not change the release mechanism of the drug from the microspheres.

\section{Conclusions}

Mucoadhesive GG microspheres were successfully prepared by ionotropic gelation with $\mathrm{Al}^{3+}$. By applying a two-level factorial design, the effects of the polymer and crosslinker concentrations on microspheres size, shape, and EE\% were demonstrated and thoroughly discussed. High polymer concentration originated more spherical and larger microspheres with higher EE\%, and the water uptake of the microspheres was no sensitive to $\mathrm{pH}$ modifications. The high mucoadhesiveness of microspheres was demonstrated in in vitro and ex vivo assays. Microspheres were able to reduce the drug release in acid and enteric $\mathrm{pH}$ compared to the free drug, demonstrating that the ionic crosslinking of GG with $\mathrm{Al}^{3+}$ allowed to produce a promising oral mucoadhesive system that controls the drug release throughout the GIT.

\section{Acknowledgements}

This work is dedicated to the memory of our dearest Professor Raul Cesar Evangelista.

The authors are thankful to CNPq (Centro Nacional de Desenvolvimento Científico e Tecnológico), to FAPESP (Fundação de Amparo à Pesquisa do Estado de São Paulo), to CAPES (Coordenação de Aperfeiçoamento de Pessoal de Nível Superior), 
and to Faculdade de Ciências Farmacêuticas de Araraquara - UNESP for providing financial and structural support to develop this work.

\section{Declaration of interest}

The authors report no declarations of interest.

\section{References}

1. Pinto JF. Site-specific drug delivery systems within the gastrointestinal tract: from the mouth to the colon. Int J Pharm 2010;395:44-52.

2. Isha C, Nimrata S, Rana AC, Surbihi G. Oral sustained release drug delivery system: an overview. Int Res J Pharm 2012;3:5762.

3. Pezzini BR, Silva MAS, Ferraz HG. Formas farmacêuticas de liberação prolongada: Sistemas monolíticos e multiparticulados. Braz J Pharm Sci 2007;43:491-502.

4. Reis AS-MJF, Pedreiro AU, Cavalcanti LN. Phosphated crosslinked pectin as a potential excipient for specific drug delivery: preparation and physicochemical characterization. Polymer Int 2010;59:127-35.

5. Torchilin VP. Drug targeting. Eur J Pharm Sci 2000;11:S81-91.

6. Philip AK, Philip B. Colon targeted drug delivery systems: a review on primary and novel approaches. Oman Med J 2010;25:79-87.

7. Friend DR. Colon-specific drug delivery. Adv Drug Deliv Rev 1991;7:149-99.

8. Sinha VR, Kumria R. Polysaccharides in colon-specific drug delivery. Int J Pharm 2001;224:19-38.

9. Carbinatto FM, De Castro AD, Cury BSF, et al. Physical properties of pectin-high amylose starch mixtures crosslinked with sodium trimetaphosphate. Int J Pharm 2012;423:281-8.

10. Meneguin AB, Cury BS, Evangelista RC. Films from resistant starch-pectin dispersions intended for colonic drug delivery. Carbohydr Polym 2014;99:140-9.

11. Osmałek T, Froelich A, Tasarek S. Application of gellan gum in pharmacy and medicine. Int J Pharm 2014;466:328-40.

12. Prezotti FG, Meneguin AB, Evangelista RC, Cury BS. Preparation and characterization of free films of high amylose/pectin mixtures cross-linked with sodium trimetaphosphate. Drug Dev Ind Pharm 2012;38:1354-9.

13. Orlu $M$, Cevher E, Araman A. Design and evaluation of colon specific drug delivery system containing flurbiprofen microsponges. Int J Pharm 2006;318:103-17.

14. Cury BS, Castro AD, Klein SI, Evangelista RC. Modeling a system of phosphated cross-linked high amylose for controlled drug release. Part 2: physical parameters, cross-linking degrees and drug delivery relationships. Int J Pharm 2009;371:8-15.

15. Bhadouriya $P$, Kumar $M$, Pathak $K$. Formulation and in vitro evaluation of prolonged release floating microspheres of atenolol using multicompartment dissolution apparatus. Drug Dev Ind Pharm 2013;39:1663-71.

16. Patil SB, Sawant KK. Chitosan microspheres as a delivery system for nasal insufflation. Colloids Surf B Biointerfaces 2011;84:384-9.

17. Vasir JK, Tambwekar K, Garg S. Bioadhesive microspheres as a controlled drug delivery system. Int $J$ Pharm 2003;255:13-32.

18. Kumari S, Bhandari A, Sharma PK. Solvent evaporation as a imposing method for microencapsulation - a review. J Drug Discov Therap 2014;2:13-20.
19. Maestrelli F, Zerrouk N, Cirri M, et al. Microspheres for colonic delivery of ketoprofen- hidroxypropyl-b-cyclodextrin complex. Eur J Pharm Sci 2008;34:1-11.

20. Schmidt C, Bodmeier R. A multiparticulate drug-delivery system based on pellets incorporated into congealable polyethylene glycol carrier materials. Int J Pharm 2001;216:9-16.

21. Jyothi NV, Prasanna PM, Sakarkar SN, et al. Microencapsulation techniques, factors influencing encapsulation efficiency. J Microencapsul 2010;27:187-97.

22. Agnihotri SA, Jawalkar SS, Aminabhavi TM. Controlled release of cephalexin through gellan gum beads: effect of formulation parameters on entrapment efficiency, size, and drug release. Eur J Pharm Biopharm 2006;63:249-61.

23. Maiti S, Ranjita S, Mandola R, et al. Al+3 ion cross-linked and acetalated gellan hydrogel network beads for prolonged release of glipizide. Carbohydr Polym 2011;85:164-72.

24. Racoviță Ş, Vasiliu S, Popa M, Luca C. Polysaccharides based on micro and nanoparticles obtained by ionic gelation and their applications as drug delivery systems. Drug Deliv Syst 2009;54:709-18.

25. Morris ER, Nishinari K, Rinaudo M. Gelation of gellan - a review. Food Hydrocoll 2012;28:373-411.

26. Picone CS, Cunha RL. Chitosan-gellan electrostatic complexes: influence of preparation conditions and surfactant presence. Carbohydr Polym 2013;94:695-703.

27. Shu XZ, Zhu KJ. Controlled drug release properties of ionically cross-linked chitosan beads: the influence of anion structure. Int J Pharm 2002;233:217-25.

28. López OV, García MA, Zaritzky NE. Film forming capacity of chemically modified corn starches. Carbohydr Polym 2008;73:573-81.

29. Narkar M, Sher P, Pawar A. Stomach-specific controlled release gellan beads of acid-soluble drug prepared by ionotropic gelation method. AAPS PharmSciTech 2010;11:267-77.

30. Cao SL, Ren XW, Zhang QZ, et al. In situ gel based on gellan gum as new carrier for nasal administration of mometasone furoate. Int J Pharm 2009;365:109-15.

31. Tayel SA, El-Nabarawi MA, Tadros MI, Abd-Elsalam WH. Promising ion-sensitive in situ ocular nanoemulsion gels of terbinafine hydrochloride: design, in vitro characterization and in vivo estimation of the ocular irritation and drug pharmacokinetics in the aqueous humor of rabbits. Int J Pharm 2013;443:293-305.

32. Emeje MO, Franklin-Ude PI, Ofoefule SI. Evaluation of the fluid uptake kinetics and drug release from gellan gum tablets containing metronidazole. Int J Biol Macromol 2010;47:158-63.

33. Reddy T, Tammishetti S. Gastric resistant microbeads of metal ion cross-linked carboxymethyl guar gum for oral drug delivery. J Microencapsul 2002;19:311-18.

34. Nokhodchi A, Tailor A. In situ cross-linking of sodium alginate with calcium and aluminum ions to sustain the release of theophylline from polymeric matrices. Farmaco 2004;59:9991004.

35. Mundargi RC, Patil SA, Agnihotri SA, Aminabhavi TM. Development of polysaccharide-based colon targeted drug delivery systems for the treatment of amoebiasis. Drug Dev Ind Pharm 2007;33:255-64.

36. Prezotti FG, Cury BS, Evangelista RC. Mucoadhesive beads of gellan gum/pectin intended to controlled delivery of drugs. Carbohydr Polym 2014;113:286-95.

37. Gohel MC, Amin AF. Formulation optimization of controlled release diclofenac sodium microspheres using factorial design. J Control Release 1998;51:115-22. 
38. Nila MV, Sudhir MR, Cinu TA, et al. Floating microspheres of carvedilol as gastro retentive drug delivery system: 3(2) full factorial design and in vitro evaluation. Drug Deliv 2014;21:110-17.

39. Dhawan S, Singla AK, Sinha VR. Evaluation of mucoadhesive properties of chitosan microspheres prepared by different methods. AAPS PharmSciTech 2004;5:e67.

40. Peterson GL. A simplification of the protein assay method of Lowry et al. which is more generally applicable. Anal Biochem 1977;83:346-56.

41. Rao KVR, Buri P. A novel in situ method to test polymers and coated microparticles for bioadhesion. Int J Pharm 1989;52:265-70.

42. Seifert DB, Phillips JA. Production of small, monodispersed alginate beads for cell imobilization. Biotechnol Prog 1997;13:562-8.

43. Chan ES, Lee BB, Ravindra P, Poncelet D. Prediction models for shape and size Ca-alginate macrobeads produced through extrusion-dripping method. J Colloid Interface Sci 2009;338:6372.

44. Cellesi F, Tirelli N, Hubbell JA. Towards a fully-synthetic substitute of alginate: development of a new process using thermal gelation and chemical cross-linking. Biomaterials 2004:25:5115-24.

45. Kaity S, Isaac J, Ghosh A. Interpenetrating polymer network of locust bean gum-poly (vinyl alcohol) for controlled release drug delivery. Carbohydr Polym 2013;94:456-67.

46. Nayak AK, Das B, Maji R. Calcium alginate/gum Arabic beads containing glibenclamide: development and in vitro characterization. Int J Biol Macromol 2012;51:1070-8.

47. Mi FL, Sung HW, Shyu SS, et al. Synthesis and characterization of biodegradable TPP/genipin co-crosslinked chitosan gel beads. Polymer 2003;44:6521-30.

48. Colombo P, Bettini R, Santi P, Peppas NA. Swellable matrices for controlled drug delivery: gel-layer behaviour, mechanisms and optimal performance. Pharm Sci Technol Today 2000;3:198-204.

49. Khare AR, Peppas NA. Swelling/deswelling of anionic copolymer gels. Biomaterials 1995;16:559-67.
50. Singh B, Bala R, Chauhan N. In vitro release dynamics of model drugs from psyllium and acrylic acid based hydrogels for the use in colon specific drug delivery. J Mater Sci Mater Med 2008;19:2771-80.

51. Simonoska Crcarevska M, Glavas Dodov M, Goracinova K. Chitosan coated Ca-alginate microparticles loaded with budesonide for delivery to the inflamed colonic mucosa. Eur J Pharm Biopharm 2008;68:565-78.

52. Mulhbacher J, Ispas-Szabo P, Mateescu MA. Cross-linked high amylose starch derivatives for drug release. II. Swelling properties and mechanistic study. Int J Pharm 2004;278:231-8.

53. Shi L, Caldwell KD. Mucin adsorption to hydrophobic surfaces. J Colloid Interface Sci 2000;224:372-81.

54. Asane GS, Nirmal SA, Rasal KB, et al. Polymers for mucoadhesive drug delivery system: a current status. Drug Dev Ind Pharm 2008;34:1246-66.

55. Gamboa JM, Leong KW. In vitro and in vivo models for the study of oral delivery of nanoparticles. Adv Drug Deliv Rev 2013;65:800-10.

56. Peppas NA, Sahlin JJ. Hydrogels as mucoadhesive and bioadhesive materials: a review. Biomaterials 1996;17:1553-61.

57. Carvalho FC, Bruschi ML, Evangelista RC, Gremião MPD. Mucoadhesive drug delivery systems. Braz J Pharm Sci 2010;46:1-17.

58. Varum FJ, Veiga F, Sousa JS, Basit AW. An investigation into the role of mucus thickness on mucoadhesion in the gastrointestinal tract of pig. Eur J Pharm Sci 2010;40:335-41.

59. Sriamornsak P, Wattanakorn N, Takeuchi H. Study on the mucoadhesion mechanism of pectin by atomic force microscopy and mucin-particle method. Carbohydr Polym 2010;79:54-9.

60. Joergensen $L$, Klösgen B, Simonsen AC, et al. New insights into the mucoadhesion of pectins by AFM roughness parameters in combination with SPR. Int J Pharm 2011;411:162-8.

61. Adams E, De Maesschalck R, De Spiegeleer B, et al. Evaluation of dissolution profiles using principal component analysis. Int J Pharm 2001;212:41-53.

62. Papadopoulou V, Kosmidis K, Vlachou M, Macheras P. On the use of the Weibull function for the discernment of drug release mechanisms. Int J Pharm 2006;309:44-50. 